\title{
ULF Doppler oscillations in the low latitude ionosphere
}

\author{
Frederick W. Menk, ${ }^{1}$ Colin L. Waters, ${ }^{1}$ and Ian S. Dunlop ${ }^{1}$ \\ Received 18 January 2007; revised 26 March 2007; accepted 13 April 2007; published 30 May 2007.
}

[1] An array of magnetometers and Doppler sounders spanning $L=1.56-2.77$ was used to examine perturbations in the daytime ionosphere driven by downgoing ULF waves. For frequencies away from the local field line resonance (FLR), the waves caused mostly vertical motions of the F-region plasma with amplitude $\sim 0.03-0.06 \mathrm{~Hz} / \mathrm{nT}$, with a phase delay of $30^{\circ}$ to $40^{\circ}$ at the ground. At the local resonant frequency and harmonics the amplitude and phase delay increased markedly. We modeled this by considering an admixture of ULF wave modes and oblique magnetic field geometry, and using actual ionospheric parameters. The model results agree well with observations when the downgoing wave mode varies smoothly from pure fast mode away from the resonance to mostly transverse mode at resonance. These results provide new information on the interaction between downgoing ULF waves and the ionospheric plasma. Citation: Menk, F. W., C. L. Waters, and I. S. Dunlop (2007), ULF Doppler oscillations in the low latitude ionosphere, Geophys. Res. Lett., 34, L10104, doi:10.1029/ 2007GL029300.

\section{Introduction}

[2] Ultra-low frequency (ULF; $\sim 1 \mathrm{mHz}-5 \mathrm{~Hz}$ ) waves propagate from the magnetosphere to the ground via the ionosphere. The wave electric and magnetic fields may drive perturbations of the ionospheric plasma during this process, causing Doppler frequency shifts in returns from HF radars [Ponomarenko et al., 2003] and HF CW sounders. The Doppler shift amplitudes are $\sim 0.1 \mathrm{~Hz} / \mathrm{nT}$ at high latitudes [Wright et al., 1997] and $\sim 0.01-0.1 \mathrm{~Hz} / \mathrm{nT}$ at low latitudes [Menk, 1992], where the smaller wave amplitudes hinder their detection.

[3] Several models have been proposed to explain such ULF Doppler oscillations. Poole et al. [1988] suggested these arise from a combination of 3 mechanisms: (1) changes in the refractive index due to variations in the longitudinal and transverse components of the wave magnetic field, requiring no bodily movement of electrons; (2) vertical motion of the electron gas driven by the eastward wave electric field (advection); and (3) compression and rarefaction of the plasma by the field-aligned component of the wave magnetic field. Production and loss of ionization is ignored at the time scales and latitudes of interest here. Poole et al. [1988] and Sutcliffe and Poole [1989] computed the amplitude and phase of Doppler oscillations in vertical incidence ionospheric records due to downgoing transverse mode waves. Zhang and Cole

\footnotetext{
${ }^{1}$ School of Mathematical and Physical Sciences, University of Newcastle, New Castle, New South Wales, Australia.
}

Copyright 2007 by the American Geophysical Union. 0094-8276/07/2007GL029300\$05.00
[1995] described a different approach that allows a mixture of incident ULF wave modes in a vertical magnetic field. This was extended by Sciffer and Waters [2002] and Sciffer et al. [2005] with an analytic formulation and a numerical model to describe mixed mode ULF wave propagation through the ionosphere for any dip angle.

[4] This paper compares new observations of ULF Doppler oscillations in the daytime low-latitude F-region with model results. Using magnetometers and Doppler sounders we examined the power and phase of ULF oscillations in the ionosphere and on the ground, distinguishing the local field line resonance (FLR) from other incident wave modes. This extends an earlier study by Marshall and Menk [1999] that considered ULF Doppler oscillations only at $L=1.83$ and for frequencies $\leq 55 \mathrm{mHz}$.

\section{Experiment and Data Processing}

[5] A purpose-built array of 5 HF CW Doppler receivers and 10 magnetometers spanning eastern Australia (see Table 1) was operated over January-April 1994. A time and frequency standard HF transmitter was located at $L=1.94$. F-region Doppler shifts could be simultaneously monitored at 5 spaced ionospheric reflection points, beneath each of which a pair of closely spaced ( $200 \mathrm{~km}$ separation) magnetometers was located. Three transmitted frequencies were monitored, providing oblique incidence reflections and Doppler shifts from essentially the same F-region altitude across the entire array.

[6] The HF Doppler receiver systems were described by Menk et al. [1995] and Marshall and Menk [1999]. These incorporated high stability frequency standards, linear frequency-to-voltage conversion, and digital data logging at $0.5 \mathrm{~Hz}$, with timing accuracy of $20-50 \mathrm{~ms}$. The frequency and phase response of each system was carefully calibrated over a passband of $2-300 \mathrm{mHz}$, and Doppler shifts $\leq 0.005 \mathrm{~Hz}$ (normalized to a frequency of $1.00 \mathrm{MHz}$ ) could be resolved. O- and X-mode HF signals could be discriminated using crossed antennas and coaxial delay lines but only the O-mode was recorded. This is typically 20-30 dB larger in amplitude than the X-mode.

[7] Each magnetometer comprised a two-component induction system [Waters et al., 1991; Menk et al., 1995] also sampled at $2.0 \mathrm{~s}$ and with identical frequency and phase response to the Doppler systems. Sensitivity (relative to the noise background) was typically $0.02 \mathrm{nT}$.

[8] Data analysis involved inspection of joint ionosphereground time series, power, cross-power and cross-phase spectra. Thus we examined the ionosphere-ground crossphase in combination with the cross-phase between magnetometer pairs, the latter allowing identification of the local FLR frequency $f_{R}$ [Waters et al., 1991] under the ionospheric reflection point. The Doppler signals were subjected to pure state vector filtering [Olson and Samson, 
Table 1. Locations of Doppler Sounders and Magnetometers ${ }^{\mathrm{a}}$

\begin{tabular}{llll}
\hline Station Name & \multicolumn{2}{l}{ Code } & Ionos- $L$ \\
\hline \multicolumn{4}{l}{ Doppler transmitter } \\
Llandilo & LAN & 1.94 & \\
& Doppler receivers & & \\
Launceston & LAI & 2.77 & 2.29 \\
Orbost & ORI & 2.29 & 2.10 \\
Clarencetown & CLI & 1.86 & 1.90 \\
Armidale & ARI & 1.74 & 1.83 \\
Bribie Is. & BRI & 1.56 & 1.73 \\
& Magnetometers & & \\
Launceston & LAU & 2.77 & \\
Orbost & ORB & 2.29 & \\
Cooma & COO & 2.15 & \\
Canberra & CAN & 2.07 & \\
Kulnura & KUL & 1.91 & \\
Newcastle & NEW & 1.86 & \\
Muswellbrook & MUS & 1.84 & \\
Armidale & ARM & 1.74 & \\
Glenn Innes & GLE & 1.69 & \\
Dalby & DAL & 1.57 & \\
\hline
\end{tabular}

${ }^{\text {a }}$ All instruments lie within $2^{\circ}$ of the $227^{\circ}$ magnetic meridian. Ionos- $L$ is the $L$ value of the ionospheric reflection point.

1979]. The state vector was set to a polarization state that best represented the azimuth and ellipticity determined over 25 days of high signal-to-noise ionosphere-ground data recorded during a separate campaign conducted in 1993. The use of this technique with relatively noisy ionospheric records is new, and greatly facilitated analysis.

\section{Observations}

[9] Correlated ionospheric Doppler oscillations and ULF pulsations were frequently observed during daylight hours, with event occurrence centered around 10 LT. The distribution of Doppler oscillations with LT and magnetic activity resembled that of ULF pulsations at the ground. Joint Doppler-ULF pulsations occurred over intervals ranging from a few wave cycles to hours, and the Doppler and magnetometer power spectra were often quite similar. The FLR and higher harmonics were readily identified in the cross-phase magnetometer and Doppler spectra.

[10] We focus on one moderately disturbed day, 12 Jan. $1994(\Sigma \mathrm{Kp}=33)$, which is representative of other days and intervals examined. The fundamental FLR and up to 3 harmonics were present in Doppler and magnetometer dynamic spectra (not shown here) between 0730-1700 LT, with frequency slowly decreasing during the day.

[11] The variation of Doppler shift amplitude with frequency and latitude over 0730-0830 LT is described in Figure 1. The $L$ values shown correspond to the ionospheric midpoint for each F-layer reflection compared with the $\mathrm{H}$ component at the nearest ground magnetometer. Vertical arrows indicate the local resonant frequency $f_{R}$ and harmonics identified from the cross-phase magnetometer spectra. For frequencies well away from resonance, the Doppler oscillation amplitude was around $0.03-0.06 \mathrm{~Hz} / \mathrm{nT}$ at all $L$ values, similar to results reported by Marshall and Menk [1999]. Amplitudes for the D components (not shown here) were similar. However, the Doppler shift amplitudes peaked sharply at $f_{R}$. This frequency decreased with increasing latitude, as expected for FLRs at these latitudes.

[12] The ionosphere-ground phase difference for this interval is shown in Figure 2, where negative phase means the ionospheric signal leads. For frequencies away from resonance the phase difference was around $-30^{\circ}$ to $-40^{\circ}$ for all $L$ values. However, this decreased sharply near $f_{R}$. A similar result was reported by Marshall and Menk [1999] at $L=1.83$, although only frequencies up to $55 \mathrm{mHz}$ could be measured. The peak in amplitude and the dip in phase identify the resonance harmonics more clearly than ground magnetometer data alone, probably because of spatial integration effects that smear out sharp amplitude and phase gradients in ground data.

\section{Comparison With Model}

[13] Modeling by Sutcliffe and Poole [1989] applies to downgoing shear Alfvén mode waves and does not predict Doppler amplitudes and phases away from $f_{R}$. We used the Sciffer et al. [2005] model to calculate the expected Doppler shifts for 12 Jan. 1994 at the $L=1.91$ location shown in Figures 1 and 2, with $f_{R}=53 \mathrm{mHz}$. Ionospheric and

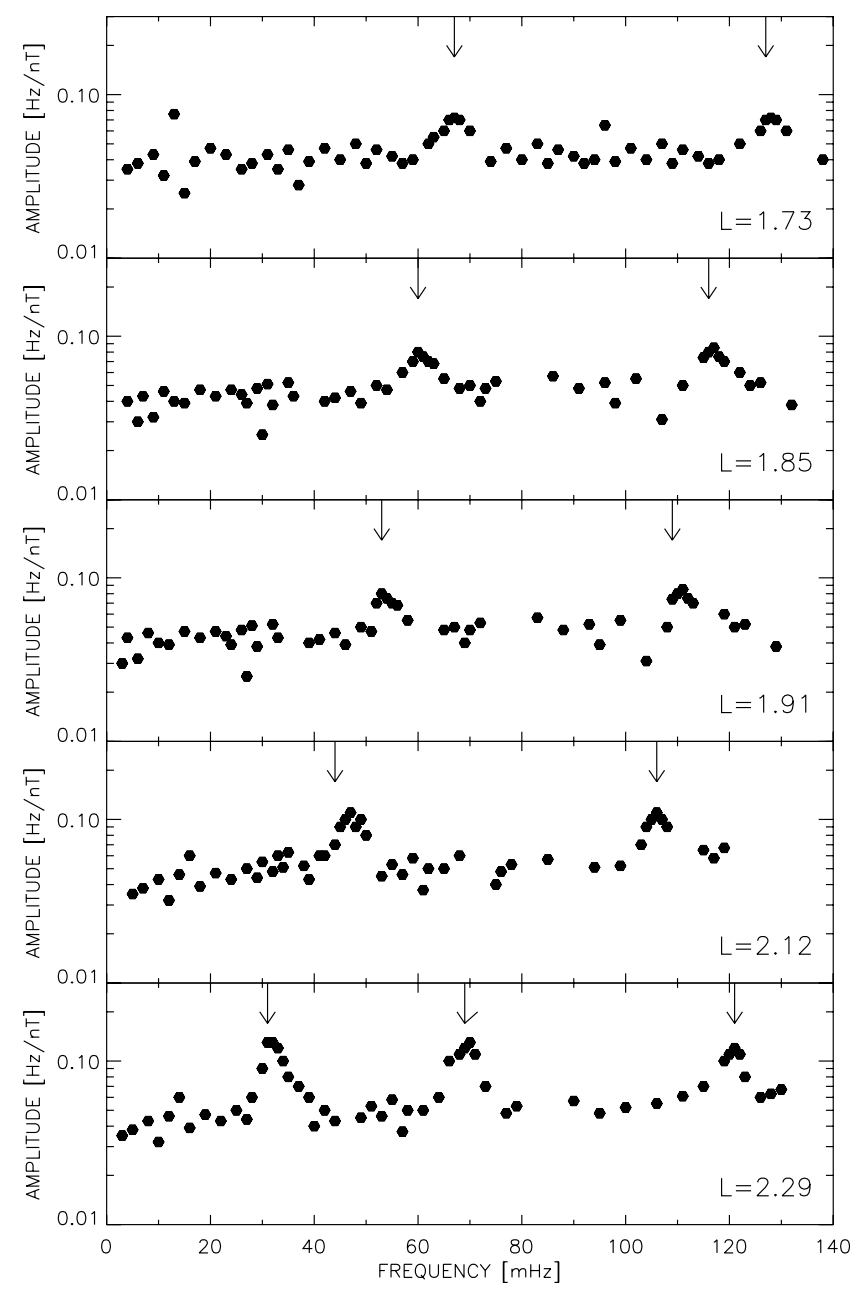

Figure 1. Normalized amplitude of ionospheric ULF Doppler oscillations on 12 Jan. 1994 as a function of frequency and $L$ value. Arrows indicate local field line resonance frequencies. 


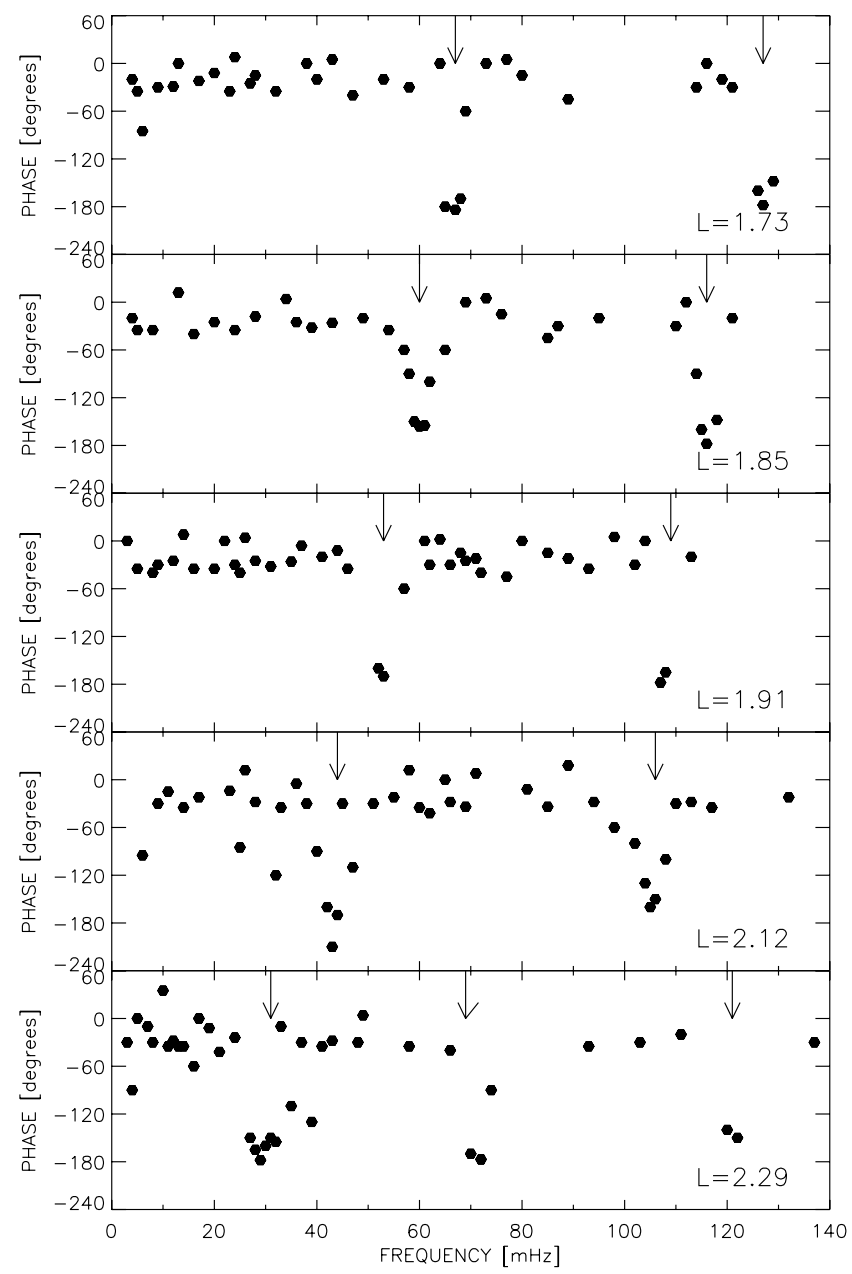

Figure 2. Ionosphere-ground phase difference for ULF Doppler oscillations on 12 Jan. 1994 as a function of frequency and $L$ value. Arrows indicate local field line resonance frequencies.

atmospheric conditions for that day were described by the IRI-95 and the MSIS90 models, and the magnetic field by the IGRF model. Other model inputs were the wave magnetic fields $b_{x}$ and $b_{y}$ observed on the ground, the observed Doppler shift (in $\mathrm{Hz} / \mathrm{nT}$ ), the magnetic dip angle and LT. Lacking direct information on the horizontal wavenumbers $k_{x}$ and $k_{y}$ for the shear Alfvén mode, we set $k_{y}=8 \times 10^{-7} \mathrm{~m}^{-1}$, corresponding to azimuthal wavenumber $m \approx 4$, similar to values reported by Ostwald et al. [1993]. We then put $k_{x}=1 \times 10^{-6} \mathrm{~m}^{-1}$, since this provided the best fit between the model results and the observations using the above parameters.

[14] We also assumed that at resonance the incoming wave mode mix at the top of the ionosphere comprises 90 $98 \%$ shear Alfvén and $10-2 \%$ fast mode. Since some damping of FLRs is expected at low latitudes, some fast mode energy is required to sustain the oscillation beyond a few cycles, as observed. Poole et al. [1988] did not include any downgoing fast mode component in their modeling. Away from the local FLR $f_{R}=53 \mathrm{mHz}$, we assumed the incoming wave is a pure fast mode at $f \leq 43 \mathrm{mHz}$ and $f \geq 63 \mathrm{mHz}$, with $k_{x}=k_{y}=8 \times 10^{-7} \mathrm{~m}^{-1}$. This $k_{y}$ value is the same as at resonance, while the wave mode mix and $k_{x}$ at other frequencies were estimated using a Gaussian fit joining the values at 43, 53 and $63 \mathrm{mHz}$.

[15] The wave magnetic field profiles for the FLR $f_{R}=$ $53 \mathrm{mHz}$ are shown in Figure 3 (top). There is negligible difference in the profiles for 90-98\% Alfvén mode. The amplitude profiles are similar to those by Poole et al. [1988] but the phases are different, mostly due to our inclusion of some downgoing fast mode component. In our case the $b_{x}$ and $b_{y}$ components experience almost no phase variation throughout the F-region, but the wave polarization azimuth changes rapidly in the E-region near $110 \mathrm{~km}$ altitude. The electric field amplitudes and phases (not shown here) do not vary greatly through the ionosphere and are similar to profiles presented by Sciffer et al. [2005].

[16] The total Doppler amplitudes and phases at $f_{R}=$ $53 \mathrm{mHz}$ arising from the combined effect of all 3 Poole et al. [1988] mechanisms are represented by the solid lines in Figure 3 (bottom), referenced to the magnetometer $\mathrm{H}$ component. The total Doppler shift maximizes near $100 \mathrm{~km}$ altitude and also near the F-region peak. We could not take measurements from the E-region, but found that the Doppler oscillations occurred most frequently and with greatest magnitude near the F-region peak. This agrees with the model predictions and observations reported by Marshall and Menk [1999]. Figure 3 also shows that at the local FLR the phase of the Doppler oscillation does not vary greatly throughout the ionosphere. The dotted lines in Figure 3 (bottom) depict the Doppler amplitude and phase for mechanism 2, representing vertical bulk motion of ionospheric plasma driven by the wave electric field. These profiles are similar to the V2 mechanism results given by Sutcliffe and Poole [1989], although the total amplitudes and phases (solid lines) are different.

[17] The model results over the range $43 \leq f \leq 63 \mathrm{mHz}$ and for $98 \%$ incident Alfvén mode are summarized in Figure 4. The model Doppler shift and phase profiles replicate the observed peak in Doppler amplitude and dip in phase at the resonant frequency at $L=1.91$ in Figures 1 and 2 . The only difference in model results for $90 \%$ Alfvén mode is a reduction in phase by $6 \%$.

\section{Discussion and Conclusions}

[18] We examined ULF wave-driven oscillations in the dayside F-region using a low-latitude array of magnetometers and Doppler sounders. Such Doppler oscillations were common occurrences, and for frequencies away from the FLR had amplitude $\sim 0.03-0.06 \mathrm{~Hz} / \mathrm{nT}$ and phase delay $\sim 30^{\circ}$ to $40^{\circ}$. However, near the local FLR and harmonics the Doppler oscillations were significantly larger and the ionosphere-ground phase difference dropped sharply to around $-160^{\circ}$ to $-180^{\circ}$.

[19] The observations are well reproduced by our model when the incoming wave mix is assumed to change smoothly from purely fast mode to $90-98 \%$ shear Alfvén mode within $10 \mathrm{mHz}$ of $f_{R}$, with $m \approx 4$ and $k_{x}$ varying with frequency. The dominant contribution to the total Doppler oscillation at resonance arises from vertical motions of the ionospheric plasma driven by the electric field of the downgoing wave. This agrees with HF radar measurements of ULF wave-driven ionospheric perturbations [Ponomarenko et al., 2003]. Our modeling also shows that 

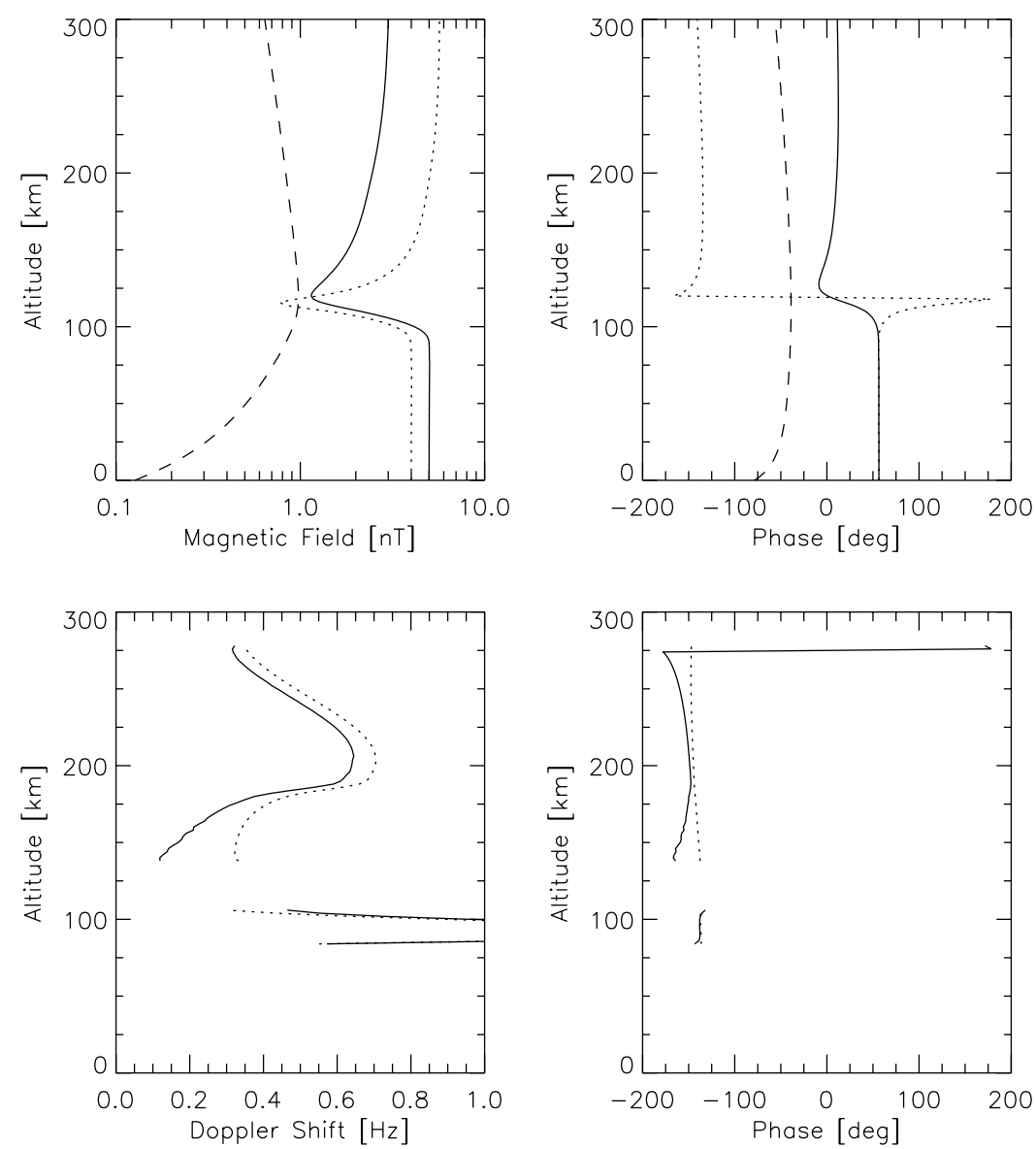

Figure 3. (top) Magnetic field and phase profiles for a downgoing shear Alfvén wave at $f_{R}=53 \mathrm{mHz}$ at $L=1.91$ on 12 Jan. 1994. $b_{x}$, solid line; $b_{y}$, dotted line; $b_{z}$, dashed line. (bottom) Resultant Doppler shift amplitude and phase profiles. Solid lines represent total Doppler shift due to all contributing mechanisms; dotted line shows effect of vertical bulk motion only (mechanism 2).

when moving away from resonance the contribution from Poole et al.'s [1988] mechanism 3 (compression) increases at high altitudes, and the changing reflection and transmission coefficients alter the phase delay. The modeled phase differences do not depend strongly on the wave mode mix at $f_{R}$ or on season (e.g. for winter conditions the shape of Figure 4 is unchanged and the minimum phase at resonance decreases by $19 \%$ ) but are quite sensitive to the choice of $k_{x}$ and $k_{y}$. Sciffer et al. [2005] discussed the variation in polarization of the downgoing wave with wavenumber and dip angle. Our numerical model assumed a onedimensional, horizontally uniform ionosphere (an approximation used in previous studies) and a detailed discussion of the effect of wave mode mix, wavenumber and ionospheric conditions on the model results are given by Waters et al. [2007].

[20] These results may be compared with measurements by Jadhav et al. [2001] of compressional ULF waves with the Oersted spacecraft as it crossed a low latitude ground station. They found that the morning ionosphere introduced a phase delay of order $60^{\circ}$ at $20 \mathrm{mHz}$, increasing to around $100^{\circ}$ delay near $65 \mathrm{mHz}$. By considering the ionospheric transmission equation for $20 \mathrm{mHz}$ fast mode waves propagating through the ionosphere in a vertical magnetic field, Kim and Takahashi [1999] deduced that around
08 MLT (the time for Figures 1 and 2) the ionosphere introduces a phase delay around $16^{\circ}$. They simply assumed that $k_{x} d \ll 1$, where $d=100 \mathrm{~km}$ is the ionospheric height. They also measured the phase difference between compressional Pc $3-4$ waves at AMPTE CCE at $L=2-3$ and an $L=1.25$ ground station, finding the ionospheric phase delay at this time was $25-47^{\circ}$ at $20 \mathrm{mHz}$.

[21] Our results also provide information on the resonance width. The widths of the Doppler amplitude and phase peaks at $L=1.91$ in Figures 1 and 2 relate to an effective resonance width $\Delta L \sim 0.1$, corresponding to a resonance $Q \approx 7$, and hence a normalized damping rate $\gamma / \omega_{r} \approx 0.07$. These widths and damping rates are considerably smaller, and the $\mathrm{Q}$ values higher, than previously reported for these latitudes [Menk et al., 2000]. This may be because the use of ionospheric measurements reduces the effects of spatial integration [e.g., Ponomarenko et al., 2001].

[22] In conclusion, our results show that downgoing ULF waves drive vertical motions of the ionospheric plasma near the F-region peak that produce Doppler oscillations in HF sounder data, and shifts in the wave phase. These effects peak sharply near the local resonance frequency, but away from resonance the ionosphere imposes an almost constant phase delay. The effects may be explained using a model of 

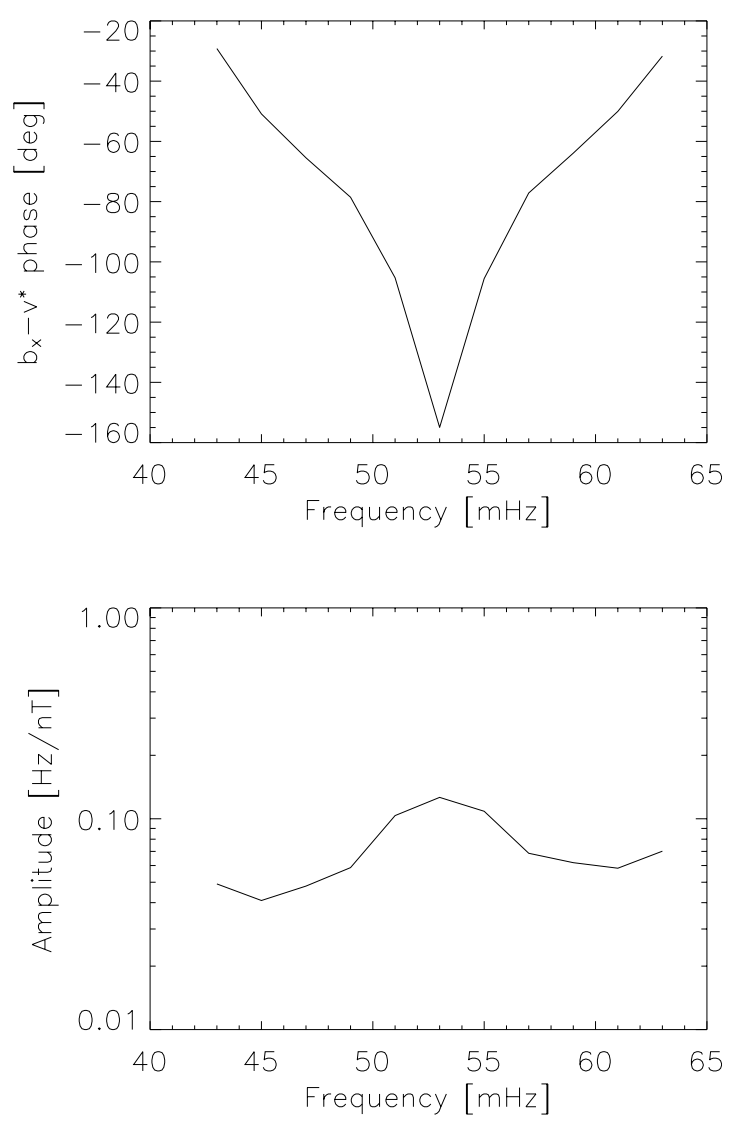

Figure 4. Modeled amplitude and phase of ULF Doppler oscillations on 12 Jan. 1994, assuming the incident wave changes from $98 \%$ Alfvén mode at $53 \mathrm{mHz}$ to pure fast modes at 43 and $63 \mathrm{mHz}$.

MHD wave propagation in which the wave mode mix varies between pure fast mode away from resonance to largely shear Alfvén mode at resonance. This provides new insight on the role and significance of field line resonances in the ionosphere and on the ground at low latitudes, including factors that influence the amplitude and phase of the signal on the ground.

[23] Acknowledgments. This work was supported by the University of Newcastle and the Australian Research Council.

\section{References}

Jadhav, G., M. Rajaram, and R. Rajaram (2001), Modification of daytime compressional waves by the ionosphere: First results from Oersted, Geophys. Res. Lett., 28, 103.

Kim, K.-H., and K. Takahashi (1999), Statistical analysis of compressional Pc3-4 pulsations observed by AMPTE CCE at $L=2-3$ in the dayside magnetosphere, J. Geophys. Res., 104, 4539.

Marshall, R. A., and F. W. Menk (1999), Low latitude observations of Pc3-4 and Pi2 geomagnetic pulsations in the ionosphere, Ann. Geophys., 17, 1397.

Menk, F. W. (1992), Characterization of ionospheric Doppler oscillations in the Pc3-4 and Pi2 magnetic pulsation frequency range, Planet. Space Sci., 40,495

Menk, F. W., R. A. Marshall, P. W. McNabb, and I. S. Dunlop (1995), An experiment to study the effects of geomagnetic fluctuations on ionospheric HF radio paths, J. Electr. Electr. Eng. Aust., 15, 325.

Menk, F. W., C. L. Waters, and B. J. Fraser (2000), Field line resonances and waveguide modes at low latitudes: 1 . Observations, J. Geophys. Res., 105,7747 .

Olson, J. V., and J. C. Samson (1979), On the detection of the polarization states of Pc micropulsations, Geophys. Res. Lett., 6, 413.

Ostwald, P. M., F. W. Menk, B. J. Fraser, C. L. Waters, and P. W. McNabb (1993), Spatial and temporal characteristics of $15-100 \mathrm{mHz}$ ULF waves recorded across a low-latitude azimuthal array, Ann. Geophys., 11, 742.

Ponomarenko, P. V., C. L. Waters, M. D. Sciffer, and B. J. Fraser (2001), Spatial structure of ULF waves: Comparison of magnetometer and Super Dual Auroral Radar Network data, J. Geophys. Res., 106, 10,509.

Ponomarenko, P. V., F. W. Menk, and C. L. Waters (2003), Visualization of ULF waves in SuperDARN data, Geophys. Res. Lett., 30(18), 1926, doi:10.1029/2003GL017757.

Poole, A. W. V., P. R. Sutcliffe, and A. D. M. Walker (1988), The relationship between ULF geomagnetic pulsations and ionospheric Doppler observations: Derivation of a model, J. Geophys. Res., 93, 14,656.

Sciffer, M. D., and C. L. Waters (2002), Propagation of ULF waves through the ionosphere: Analytic solutions for oblique magnetic fields, J. Geophys. Res., 107(A10), 1297, doi:10.1029/2001JA000184

Sciffer, M. D., C. L. Waters, and F. W. Menk (2005), A numerical model to investigate the polarisation azimuth of ULF waves through an ionosphere with oblique magnetic fields, Ann. Geophys., 23, 3457, SRef-ID:14320576/ag/2005-23-3457.

Sutcliffe, P. R., and A. W. V. Poole (1989), Ionospheric Doppler and electron velocities in the presence of ULF waves, J. Geophys. Res., 94, 13,505 .

Waters, C. L., F. W. Menk, and B. J. Fraser (1991), The resonance structure of low latitude Pc3 geomagnetic pulsations, Geophys. Res. Lett., 18 2293

Waters, C. L., T. K. Yeoman, M. D. Sciffer, P. V. Ponomarenko, and D. M. Wright (2007), Modulation of radio frequency signals by ULF waves, Ann Geophys, in press.

Wright, D. M., T. K. Yeoman, and P. J. Chapman (1997), High-latitude HF Doppler observations of ULF waves: 1 . Waves with large spatial scale sizes, Ann. Geophys., 15, 1548

Zhang, D. Y., and K. D. Cole (1995), Formulation and computation of hydromagnetic wave penetration into the equatorial ionosphere and atmosphere, J. Atmos. Terr. Phys., 57, 813.

I. S. Dunlop, F. W. Menk, and C. L. Waters, School of Mathematical and Physical Sciences, University of Newcastle, University Drive, Callaghan, Newcastle, NSW 2308, Australia. (fred.menk@newcastle.edu.au; colin. waters@newcastle.edu.au) 